ARTICLE

DOI: $10.1038 / \mathrm{s} 41467-018-06247-5$

\title{
The ZZ-type zinc finger of ZZZ3 modulates the ATAC complex-mediated histone acetylation and gene activation
}

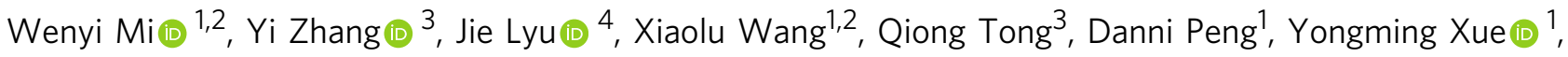
Adam H. Tencer ${ }^{3}$, Hong Wen ${ }^{1,2}$, Wei Li (i] ${ }^{4}$, Tatiana G. Kutateladze (i) ${ }^{3}$ \& Xiaobing Shi (i) ${ }^{1,2}$

Recognition of histones by epigenetic readers is a fundamental mechanism for the regulation of chromatin and transcription. Most reader modules target specific post-translational modifications on histones. Here, we report the identification of a reader of histone $\mathrm{H} 3$, the ZZ-type zinc finger (ZZ) domain of ZZZ3, a subunit of the Ada-two-A-containing (ATAC) histone acetyltransferase complex. The solution NMR structure of the $Z Z$ in complex with the $\mathrm{H} 3$ peptide reveals a unique binding mechanism involving caging of the $\mathrm{N}$-terminal Alanine 1 of histone $\mathrm{H} 3$ in an acidic cavity of the $\mathrm{ZZ}$ domain, indicating a specific recognition of $\mathrm{H} 3$ versus other histones. Depletion of ZZZ3 or disruption of the ZZ-H3 interaction dampens ATAC-dependent promoter histone H3K9 acetylation and target gene expression. Overall, our study identifies the $\mathrm{ZZ}$ domain of $\mathrm{ZZZ3}$ as a histone $\mathrm{H} 3$ reader that is required for the ATAC complex-mediated maintenance of histone acetylation and gene activation.

\footnotetext{
${ }^{1}$ Department of Epigenetics and Molecular Carcinogenesis, Center for Cancer Epigenetics, The University of Texas MD Anderson Cancer Center, Houston, Texas 77030, USA. ${ }^{2}$ Center for Epigenetics, Van Andel Research Institute, Grand Rapids, Michigan 49503, USA. ${ }^{3}$ Department of Pharmacology, University of Colorado School of Medicine, Aurora, Colorado 80045, USA. ${ }^{4}$ Dan L. Duncan Cancer Center, Department of Molecular and Cellular Biology, Baylor College of Medicine, Houston, Texas 77030, USA. These authors contributed equally: Wenyi Mi, Yi Zhang and Jie Lyu. Correspondence and requests for materials should be addressed to T.G.K. (email: tatiana.kutateladze@ucdenver.edu) or to X.S. (email: xiaobing.shi@vai.org)
} 
$\mathrm{P}$ ost-translational modifications (PTMs) of histones, such as acetylation and methylation, play an important role in modulating chromatin structure and all chromatinassociated processes, including gene transcription ${ }^{1,2}$. Acetylation on histones is normally associated with gene activation, whereas methylation is linked to either gene activation or repression, depending on the specific residues being modified. In general, methylation on histone $\mathrm{H} 3 \mathrm{~K} 4, \mathrm{H} 3 \mathrm{~K} 36$, and $\mathrm{H} 3 \mathrm{~K} 79$ is enriched in actively transcribed genes, and di- and trimethylation on $\mathrm{H} 3 \mathrm{~K} 9$, $\mathrm{H} 3 \mathrm{~K} 27$, and H4K20 occupies silent genes ${ }^{3}$. The principal function of histone acetylation and methylation is to recruit or repel "reader" proteins that recognize the small moieties on modified histone residues and transduce the epigenetic signals at chromatin to distinct biological outcomes ${ }^{4}$. Studies from many laboratories have identified a number of readers selective to specific histone modifications, for example, the chromodomain (CHD) and the plant homeodomain (PHD) zinc finger recognizes methylation on distinct lysine residues of histones ${ }^{5-8}$, whereas the bromodomain (BRD) and the YEATS domain bind to acetylated histones ${ }^{9,10}$. To date, almost all known histone readers are specific or sensitive to certain histone PTMs; however, a versatile reader that can accommodate common modifications on histones has yet to be discovered.

ZZZ3 (zinc finger ZZ-type containing 3) is a core subunit of the ATAC complex, a conserved metazoan histone acetyltransferase (HAT) complex ${ }^{11,12}$. In mammals, ATAC contains one of the two highly related, mutually exclusive catalytic subunits, GCN5 or PCAF, which are shared with another major HAT complex, the Spt-Ada-Gcn5-Acetyltransferase (SAGA) complex ${ }^{13,14}$. While biological activities of the SAGA components have been extensively studied ${ }^{15,16}$, the functions of most of the ATAC complex subunits, including ZZZ3, remain largely unknown.

Here, we report the identification of the ZZ-type zinc finger (ZZ) as a family of histone $\mathrm{H} 3$ reader. The solution NMR structure of the $\mathrm{ZZ}$ domain of ZZZ3 in complex with the H3 peptide reveals a unique binding mechanism involving caging of the N-terminal Alanine 1 of histone $\mathrm{H} 3$ in an acidic cavity of the $\mathrm{ZZ}$ domain. The $\mathrm{ZZ}$ domain specifically binds to histone $\mathrm{H} 3$ tail, making contacts mainly with the first four amino acids of histone H3. Surprisingly, this recognition is insensitive to methylation on H3K4 or H3R2, whereas acetylation on H3K4 moderately enhances the binding. The recognition of histone $\mathrm{H} 3$ by $\mathrm{ZZ}$ is essential for chromatin occupancy of ZZZ3 and functions of the ATAC complex in cells. Depletion of ZZZ3 or disruption of the ZZ-H3 interaction dampens ATAC-dependent promoter histone $\mathrm{H} 3 \mathrm{~K} 9$ acetylation and expression of target genes, including the ribosomal protein encoding genes. Overall, our study identified the $\mathrm{ZZ}$ domain of $\mathrm{ZZZ3}$ as a versatile histone $\mathrm{H} 3$ reader that is required for the ATAC complex-mediated maintenance of histone acetylation and gene activation .

\section{Results}

The $\mathrm{ZZ}$ domain is a histone $\mathrm{H3}$-recognizing module. $\mathrm{ZZZ3}$ contains two conserved domains, a putative DNA-binding Swi3Ada2-NCOR-TAFIIB (SANT) domain and a ZZ of unknown function (Fig. 1a). Screening for novel readers by a homemade histone peptide array, we found that the ZZ of human ZZZ3 binds to the N-terminal tail of histone $\mathrm{H} 3$ in a methylationindependent manner (Fig. 1b). In vitro histone-binding experiments (Fig. 1c) and histone peptide pulldowns (Fig. $1 \mathrm{~d}$ and Supplementary Fig. 1a) further demonstrated that ZZZ3 ZZ specifically recognizes the $\mathrm{H} 3$ tail, but not other regions of $\mathrm{H} 3$, nor other histones. The $\mathrm{N}$-terminal eight amino acids of $\mathrm{H} 3$ were sufficient for ZZZ3 binding; removal of the first four amino acids of the $\mathrm{H} 3$ tail completely abolished the binding (Fig. 1e), suggesting that the very N-terminal end of $\mathrm{H} 3$ is critical for the ZZZ3-H3 interaction. Unlike other known H3-binding modules, such as the WD40 repeats of WDR5 and the first PHD finger (PHD1) of RBP2 that are sensitive to methylation on H3R2 and $\mathrm{H} 3 \mathrm{~K} 4$, respectively ${ }^{17-19}$, the ZZZ3 ZZ-H3 interaction was not affected by methylation on these residues (Fig. 1d-f). In contrast, acetylation on $\mathrm{H} 3 \mathrm{~K} 4$ moderately enhanced ZZZ3 ZZ binding to H3 (Fig. 1g and Supplementary Fig. 1b), implying a novel mode of $\mathrm{H} 3$ recognition.

To determine whether $\mathrm{H} 3$ binding is a common feature of $\mathrm{ZZ}$ domains, we cloned 16 zinc fingers of all the human $\mathrm{ZZ}$ proteins that reside in, or shuttle into the nucleus ${ }^{13}$, and tested their $\mathrm{H} 3$ binding activity in pull-down experiments with histone peptides and full-length histones. We found that 9 of the $16 \mathrm{ZZ}$ domains could bind to histone H3 tail (Supplementary Fig. 1c-e), suggesting that $\mathrm{H} 3$ binding has evolved as a common function of $\mathrm{ZZ}$ domains within a subset of the protein family (Fig. 1h).

Molecular basis of recognition of $\mathrm{H3}$ by the $\mathrm{ZZZ3} \mathrm{ZZ}$ domain. To define the molecular mechanism by which ZZZ3 recognizes histone $\mathrm{H} 3$, we determined the solution structure of the ZZZ3 ZZ domain (aa 816-874) in complex with an unmodified histone $\mathrm{H} 3$ tail peptide (aa 1-12) by NMR spectroscopy. The structural ensemble data were well defined throughout the protein and the four $\mathrm{N}$-terminal residues of the $\mathrm{H} 3$ peptide (Supplementary Fig. 2a and Supplementary Table 1). The structure shows a crossbrace topology fold, with a central three-stranded antiparallel $\beta$ sheet (aa 847-849, 835-838, and 869-871), a short $\alpha$-helix (aa 851-854), and a small two-stranded $\beta$-sheet (aa 817-818 and 831-832) at the N-terminus (Fig. 2a). The histone peptide is bound in an extended conformation, forming a short twostranded antiparallel $\beta$-sheet with the residues $821-823$ of $\mathrm{ZZ}$. The four $\mathrm{N}$-terminal residues of the $\mathrm{H} 3$ peptide are in direct contact with ZZ, while the rest of the tail is unstructured and solvent-exposed (Fig. $2 \mathrm{~b}$ and Supplementary Fig. 2b). Ala1 of the $\mathrm{H} 3$ peptide inserts deeply into the negatively charged cavity of the protein, and its amino-terminal $\mathrm{NH}_{3}{ }^{+}$group is restrained through interactions with $\mathrm{ZZ}$, specifically the side-chain carboxyl groups of D824 and D848 and the carbonyl group of K822 (Fig. 2c).

Consistent with the structural results, blocking the aminoterminal $\mathrm{NH}_{3}+$ group of $\mathrm{H} 3$ Ala1 by an acetyl group or biotin impeded ZZZ3 ZZ-H3 interaction (Supplementary Fig. 2c); and deletion of the first two amino acids (Ala1-Arg2) of the H3 peptide completely abolished its interaction with all $\mathrm{ZZ}$ domains, as observed in peptide pulldown (Supplementary Fig. 1d) and NMR titration experiments (Supplementary Fig. 2d). Tryptophan fluorescence experiments revealed a $K_{\mathrm{d}}$ of $29 \mu \mathrm{M}$ for ZZZ3 ZZ association with the $\mathrm{H} 3$ peptide (Fig. 2d). Substitution of D824 or D848 in the $\mathrm{ZZ}$ domain with an alanine completely disrupted its interaction with the $\mathrm{H} 3$ peptide (Fig. 2d and Supplementary Fig. 2e, f). Importantly, all the H3-binding ZZ domains contain an invariable aspartate (D848 in ZZZ3) along with an aspartate (D824 in ZZZ3) or asparagine (in p300 and CBP ZZs) (Supplementary Fig. $2 \mathrm{~g}$ and the accompanied manuscript), suggesting that recognition of Ala1 of $\mathrm{H} 3$ through the acidic cage is likely a common mechanism of the $\mathrm{ZZ}$ readers. However, simply introducing acidic residues into the non-H3-binding $\mathrm{ZZ}$ domains of TADA2A and TADA2B did not render them $\mathrm{H} 3$ binding activity (Supplementary Fig. 2h), suggesting that interactions other than with the acidic residues are also required. In line with this, an N-terminal Ala1 added to a non-H3-like peptide (AGSGSG) was insufficient to bind the ZZZ3 ZZ domain (Supplementary Fig. 2i). 
a
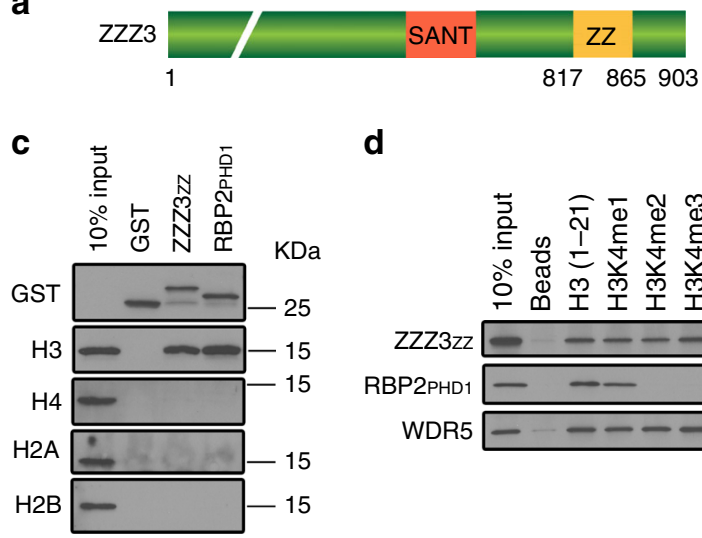

d

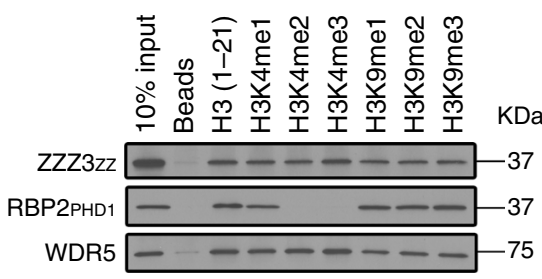

b

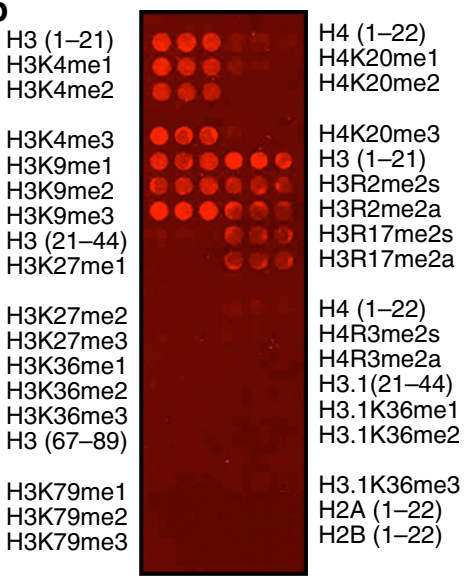

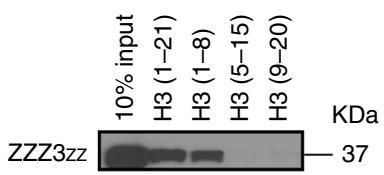

f

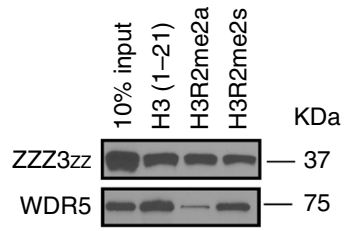

g

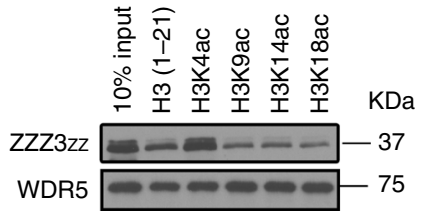

h

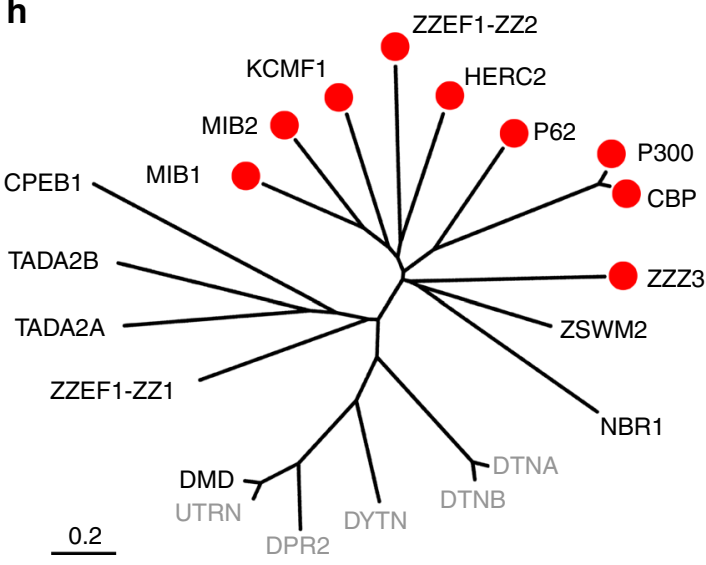

Fig. 1 The $Z Z$ domain of $Z Z Z 3$ recognizes histone $H 3$ tail. a Schematic representation of $Z Z Z 3$ protein structure. The amino acid numbers of $Z Z$ domain and the full-length protein are shown. $\mathbf{b}$ Histone peptide microarray probed with GST-ZZZZ ZZ domain. Each peptide has three replicates. $\mathbf{c}$ Western blot analysis of GST pulldowns of GST-ZZZ3 ZZ with calf thymus histones. Pulldown of GST-RBP2 PHD1 is shown as a control. $\mathbf{d}$ Western blot analysis of peptide pull-down experiments of GST-ZZZ3 ZZ domain with the indicated histone H3 peptides. Peptide pulldowns of RBP2 PHD1 and WDR5 are shown for comparison. e-g Western blot analysis of peptide pulldown experiments of GST-ZZZ3 ZZ domain with the indicated H3 peptides. Peptide pulldowns of WDR5 are shown for comparison. $\mathbf{h}$ Phylogenetic tree presentation of the $\mathrm{H} 3$-binding activity of human $\mathrm{ZZ}$ proteins. The red dot indicates binding with $\mathrm{H} 3$ (Supplementary Fig. 1c-e). The cytoplasmic ZZ proteins (gray) are not tested in this study

H3K4 acetylation moderately enhances the ZZZ3-H3 binding. Although methylation has no apparent effect on the binding of ZZZ3 ZZ to the $\mathrm{H} 3$ peptide, acetylation on $\mathrm{H} 3 \mathrm{~K} 4$ moderately enhances the interaction, as observed in peptide arrays, pulldowns, and NMR titrations (Fig. 1b-d-g, Supplementary Figs $1 \mathrm{~b}$ and $3 \mathrm{a}, \mathrm{b})$. Consistent with these observations, tryptophan fluorescence experiments showed an $\sim 3$-fold enhancement for $\mathrm{ZZ}$ association with the $\mathrm{H} 3 \mathrm{~K} 4 \mathrm{ac}$ peptide $\left(K_{\mathrm{d}}=8.2 \mu \mathrm{M}\right)$ over its association with the unmodified $\mathrm{H} 3$ peptide $\left(K_{\mathrm{d}}=29 \mu \mathrm{M}\right)$ (Fig. 2d and Supplementary Fig. 3c). Microscale thermophoresis (MST) binding assays further corroborated the threefold enhancement (Fig. 2e and Supplementary Fig. 3d).

To understand the enhancement of binding to acetylated $\mathrm{H} 3 \mathrm{~K} 4$, we determined the solution structure of the ZZZ3 ZZ domain in complex with an $\mathrm{H} 3 \mathrm{~K} 4 \mathrm{ac}$ peptide (aa 1-8) using NMR spectroscopy (Supplementary Fig. $3 e$ and Supplementary Table 1). Structural comparison of the H3-bound and H3K4ac-bound ZZ revealed that $\mathrm{H} 3 \mathrm{~K} 4$ lays in a hydrophobic groove formed by F821, G820, and V819 in both complexes; however, the mean position of the acetylated side chain of Lys 4 in the ensemble of the NMR structures is located closer to the side chain of F821 of ZZ compared to the mean position of the unmodified Lys4 (Fig. $2 \mathrm{f}$ and Supplementary Fig. 3f). We speculated that the hydrophobic interaction between the neutral side chain of acetylated Lys 4 and the aromatic ring of ZZ F821 might account for the increase in binding affinity. To test this hypothesis, we substituted F821 for an alanine and examined binding of the F821A mutant to the $\mathrm{H} 3 \mathrm{~K} 4 \mathrm{ac}$ and unmodified $\mathrm{H} 3$ peptides using several orthogonal methods. We found that alanine substitution substantially decreases $\mathrm{ZZ}$ binding to either the unmodified or the Lys4-acetylated $\mathrm{H} 3$ peptides (Fig. 2g), and that the F821A mutant associates equally with both peptides in NMR titration and tryptophan fluorescence assays (Fig. 2d and Supplementary $3 \mathrm{~b}-\mathrm{h})$. The acetylation-dependent enhancement of $\mathrm{H} 3$ binding by $\mathrm{ZZ}$ is reminiscent to the acetylation- or methylation-dependent enhancement in binding activity of other $\mathrm{H} 3$ readers. Much like the ZZZ3 ZZ domain, the DPF module of DPF3b or MORF binds 3-fold tighter to H3K14ac than to the unmodified $\mathrm{H} 3$ peptide ${ }^{20-23}$. Structural comparison reveals that although the overall $\mathrm{ZZ}$ and $\mathrm{DPF} 3 \mathrm{~b}^{23}$ exhibit distinct mechanisms for the recognition of the histone $\mathrm{H} 3$ tail, in both cases, the hydrophobic character of the acetyllysine-binding sites and the presence of aromatic residues most likely facilitate binding to a hydrophobic acetylated lysine species (Supplementary Fig. 4a, b). 
a

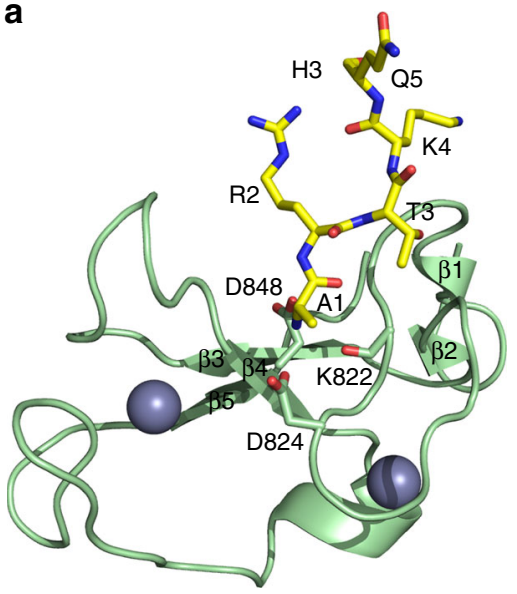

C

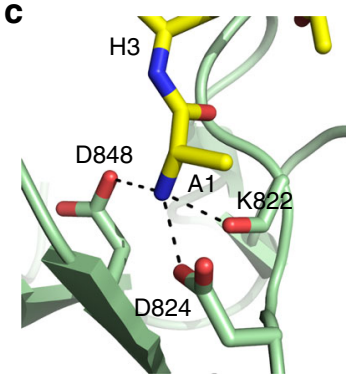

e

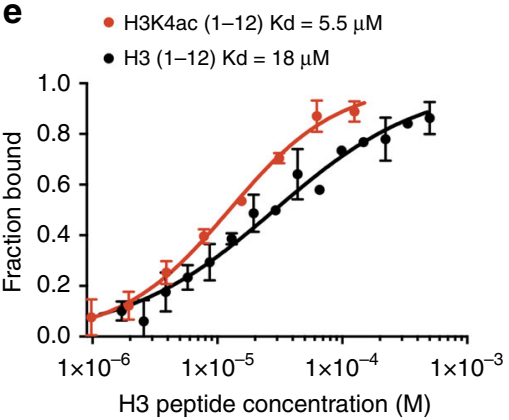

b

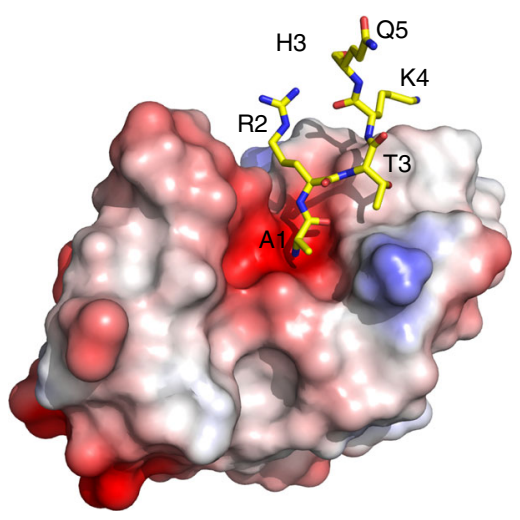

d

\begin{tabular}{ccc}
\hline ZZ domain & $\mathrm{H} 3(1-12)$ peptide & $\mathrm{Kd}(\mu \mathrm{M})$ \\
\hline \multirow{2}{*}{ WT } & $\mathrm{H} 3$ & $29 \pm 7$ \\
& $\mathrm{H} 3 \mathrm{~K} 4 \mathrm{me3}$ & $22 \pm 6$ \\
& $\mathrm{H} 3 \mathrm{~K} 4 \mathrm{ac}$ & $8.2 \pm 2.4$ \\
\hline \multirow{2}{*}{ F821A } & $\mathrm{H} 3$ & $271 \pm 33$ \\
& $\mathrm{H} 3 \mathrm{~K} 4 \mathrm{ac}$ & $294 \pm 83$ \\
\hline \multirow{2}{*}{ D824A } & $\mathrm{H} 3$ & No binding $^{*}$ \\
& $\mathrm{H} 3 \mathrm{~K} 4 \mathrm{ac}$ & No binding $^{*}$ \\
\hline D848A & $\mathrm{H} 3$ & No binding $^{*}$ \\
\hline
\end{tabular}

f

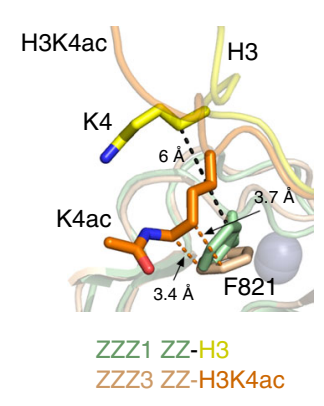

g

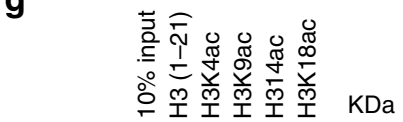

WT ------37

N F821A $=37$

N D824A $=37$

D848A $=37$

Fig. 2 Structure of the $Z Z Z 3 Z Z$ domain in complex with $H 3$ peptides. a Ribbon diagram of the $Z Z Z 3 Z Z Z_{816-874}$ domain (green) in complex with the histone $\mathrm{H} 3$ peptide (yellow). $\mathbf{b}$ Electrostatic potential surface of $Z Z Z 3 Z Z$ in complex with the histone $\mathrm{H} 3$ peptide (yellow stick). A positive electrostatic potential is colored in blue and negative in red. c Zoom-in view of the histone H3 Ala1 binding site. ZZZ3 ZZ domain is shown as a green ribbon and the histone H3 peptide as a yellow stick. The dash lines indicate short distances. d Binding affinities of WT ZZZZ ZZ and mutants for the indicated histone peptides measured by tryptophan fluorescence. The experiments were carried out in triplicates. *: measured by NMR. e Binding curves used to determine the $K_{d}$ values by MST. $\mathbf{f} Z$ Zoom-in view of the mean position of Lys 4 in each ensemble of 20 NMR structures of the complexes of ZZZ3 ZZ with unmodified H3 peptide and with $\mathrm{H} 3 \mathrm{~K} 4 \mathrm{ac}$ peptide are superimposed. $\mathbf{g}$ Western blot analysis of histone peptide pulldowns of wild-type (WT) ZZZ3 ZZ and the indicated point mutants

Similarly, a single aromatic residue in the PHD finger of CHD4 ${ }^{24}$ provides favorable hydrophobic and cation-pi contacts with H3K9me3 (Supplementary Fig. 4c).

ZZZ3 is critical for ATAC-dependent histone acetylation. The catalytic subunit GCN5 or PCAF in the ATAC complex has been shown to catalyze acetylation of histone $\mathrm{H} 3$ on $\mathrm{H} 3 \mathrm{~K} 9, \mathrm{H} 3 \mathrm{~K} 14$, and $\mathrm{H} 3 \mathrm{~K} 4^{11,12,25}$. Because ZZZ3 is a stoichiometric component of the ATAC complex, we speculated that recognition of $\mathrm{H} 3$ by the ZZZ3 ZZ domain may facilitate substrate association of ATAC, thus promoting $\mathrm{H} 3$ acetylation (Fig. 3a). To test this hypothesis, we carried out in vitro HAT assays of purified ATAC complex from cultured cells using histone peptides and reconstituted unmodified nucleosomes as substrates. We found that the purified ATAC complex shows robust acetylation activity on $\mathrm{H} 3 \mathrm{~K} 9$ and relatively weak activity on $\mathrm{H} 3 \mathrm{~K} 4$ (Fig. 3b, c). Importantly, deletion of the first two amino acids from histone $\mathrm{H} 3$ greatly reduced the HAT activity of ATAC on both the histone peptide and reconstituted nucleosomes. Compared with the unmodified $\mathrm{H} 3$ peptide, the Lys4-acetylated $\mathrm{H} 3$ peptide is a better substrate for ATAC (Supplementary Fig. 5a). Furthermore, substitution of F821 and D824 of ZZZ3 to an alanine substantially reduced the HAT activity of ATAC on $\mathrm{H} 3$ without affecting the complex integrity (Fig. 3d and Supplementary Fig. 5b, c). Together, these results suggest that binding of $\mathrm{H} 3$ by $\mathrm{ZZZ3}$ is required for efficient $\mathrm{H} 3$ acetylation by the ATAC complex.

Consistent with the in vitro data, depletion of ZZZ3 by shRNA-mediated knockdown (KD) in H1299 and A549 lung adenocarcinoma cell lines resulted in reduction in global $\mathrm{H} 3 \mathrm{~K} 9$ and H3K4 acetylation levels (Fig. 3e). This effect is not due to the 
a

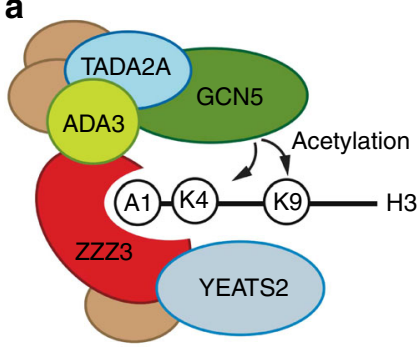

b

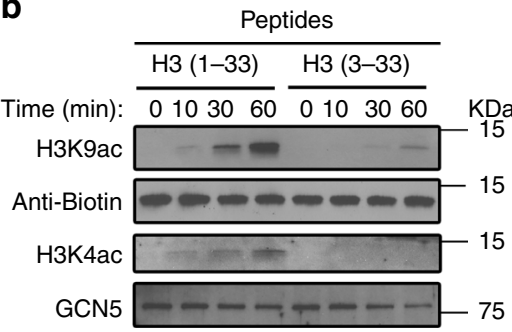

C

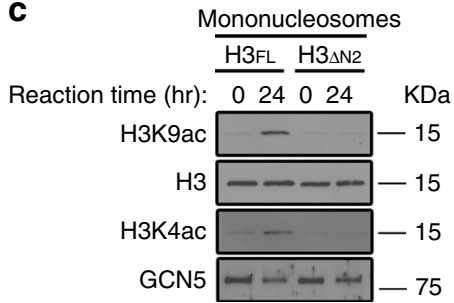

d

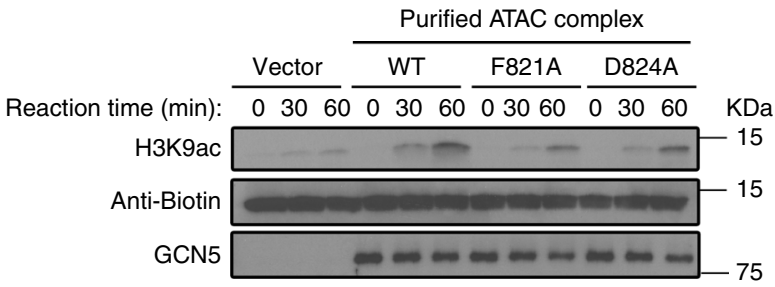

e
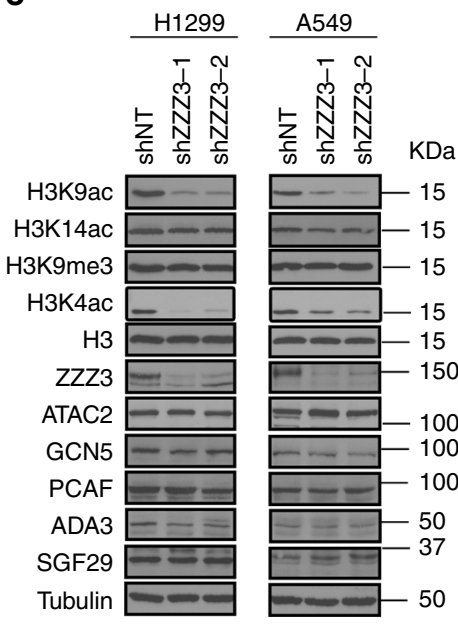

f

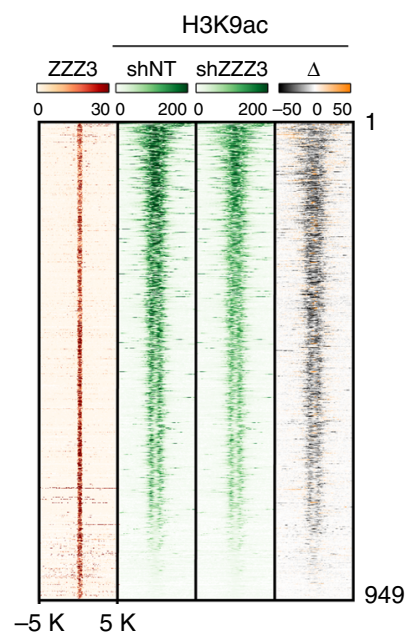

Fig. 3 Recognition of $\mathrm{H} 3$ by ZZZ3 is critical for ATAC-dependent histone acetylation. a Schematic of ZZZ3 and the ATAC complex in recognition and acetylation of histone H3 tail. b, c Deletion the first two amino acids (Ala1-Arg2) of H3 abolishes the HAT activity of the ATAC complex in vitro. Western blot analysis of HAT assays of purified ATAC complex using the H3 (1-33) or H3 (3-33) peptides (b), or reconstituted mononucleosome containing fulllength $\mathrm{H} 3$ or $\mathrm{H} 3 \mathrm{~N} \Delta 2$ proteins (c) as substrates. Biotinylated peptides and $\mathrm{H} 3$ are shown as loading controls. $\mathbf{d}$ Point mutations of $\mathrm{ZZ}$ domain attenuate the HAT activity of the ATAC complex in vitro. Western blot analysis of HAT assays of purified ATAC complexes containing WT or mutant ZZZ3 using H3 (1-33) peptide as a substrate. Biotinylated peptides are shown as loading controls. e ZZZ3 KD reduces global H3K9ac and H3K4ac levels in cells. Western blot analysis of the indicated histone modifications and protein levels of ATAC subunits in control (shNT) and ZZZ3 KD (shZZZ3) cells. H3 and tubulin are used as loading controls. $\mathbf{f}$ Heatmap profiles of ZZZ3 in H1299 cells, H3K9ac in control (shNT) and ZZZ3 KD (shZZZ3) cells, and difference of H3K9ac $(\Delta$, shZZZ3-shNT). The color key represents the signal density. Gray indicates a reduction in ZZZ3 KD cells compared to the control cells

loss of complex integrity as the protein levels of GCN5/PCAF and other ATAC complex components remained unchanged. ChIP experiments followed by high-throughput sequencing (ChIP-seq) revealed that the reduction of $\mathrm{H} 3 \mathrm{~K} 9$ acetylation is specific to ZZZ3-occupied genes (described below), as the H3K9ac levels on non-ZZZ3-occupied genes are largely not affected by ZZZ3 KD (Fig. 3f and Supplementary Fig. 5d). ChIP followed by quantitative real-time PCR (qPCR) analysis in ZZZ3 KD cells revealed that depletion of $\mathrm{ZZZ3}$ reduces $\mathrm{H} 3 \mathrm{~K} 9 \mathrm{ac}$ and $\mathrm{H} 3 \mathrm{~K} 4 \mathrm{ac}$ levels on ZZZ3 target genes (Supplementary Fig. 6a-c). This reduction is likely due to the loss of ATAC occupancy on chromatin, as the binding of YEATS2, an ATAC-specific subunit, on ATAC target genes was substantially reduced upon ZZZ3 KD, whereas the occupancy of the SAGA subunit SPT20 and H3K9ac levels on SAGA target genes was not affected (Supplementary Fig. 6d-f). Depletion of either GCN5 or PCAF considerably reduced target gene $\mathrm{H} 3 \mathrm{~K} 9$ acetylation levels, and double $\mathrm{KD}$ showed an additive effect (Supplementary Fig. 6g, h), suggesting that likely both GCN5 and PCAF contribute to ATAC-dependent maintenance of histone acetylation.

ZZZ3 is required for ATAC complex-dependent gene expression. To determine the functional importance of $\mathrm{H} 3$ recognition by the $\mathrm{ZZ}$ domain in cells, we first investigated its role in ZZZ3 binding to chromatin. We performed ChIP-seq experiments in H1299 cells to assess the genome-wide occupancy of ZZZ3 and its correlation with the ATAC complex and active transcriptionassociated histone marks (H3K4me3, H3K4ac, and $\mathrm{H} 3 \mathrm{~K} 9 \mathrm{ac}$ ). Using a validated ChIP-grade anti-ZZZ3 antibody ${ }^{26}$, we identified 949 ZZZ3-occupied peaks that are strongly enriched in promoters ( $\pm 3 \mathrm{~kb}$ of the transcription start site, TSS) (Supplementary Fig. 7a and Supplementary Data 1). We determined genome-wide occupancy of Flag-YEATS2 (Supplementary Data 1) by Flag ChIP-seq and used it as a surrogate of the ATAC complex. We found that although Flag ChIP-seq yielded many more peaks, the majority of the ZZZ3 peaks overlap with Flag-YEATS2 peaks (Supplementary Fig. 7b). Furthermore, more than three-quarters of ZZZ3 peaks co-localize with $\mathrm{H} 3 \mathrm{~K} 4 \mathrm{ac}, \mathrm{H} 3 \mathrm{~K} 9 \mathrm{ac}$, and $\mathrm{H} 3 \mathrm{~K} 4 \mathrm{me} 3$ occupied peaks (Fig. 4a and Supplementary Fig. 7c), and all peaks are enriched at gene promoters (Fig. $4 \mathrm{~b}$ and Supplementary Fig. 7d, e).

We then performed RNA-seq analysis in ZZZ3 KD cells to identify ZZZ3 regulated genes. We used two independent shRNAs targeting ZZZ3 to minimize the off-target effect of shRNAs. We identified 1333 genes downregulated and 1836 genes upregulated in both shRNA KD cells compared with the cells treated with a non-targeting shRNA (Supplementary Fig. 8a-c and Supplementary Data 2), with the downregulated genes strongly enriched in the pathways of ribosome biogenesis (Supplementary Fig. 8d). Co-analysis of the ZZZ3 ChIP-seq and 
a

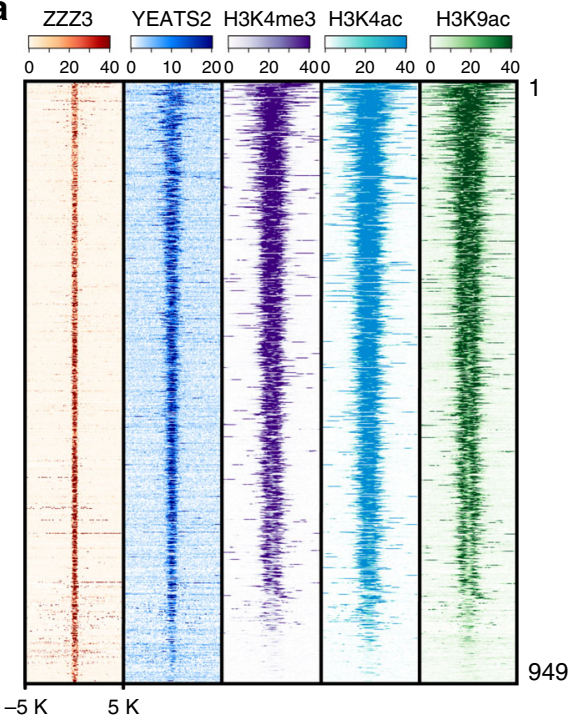

b

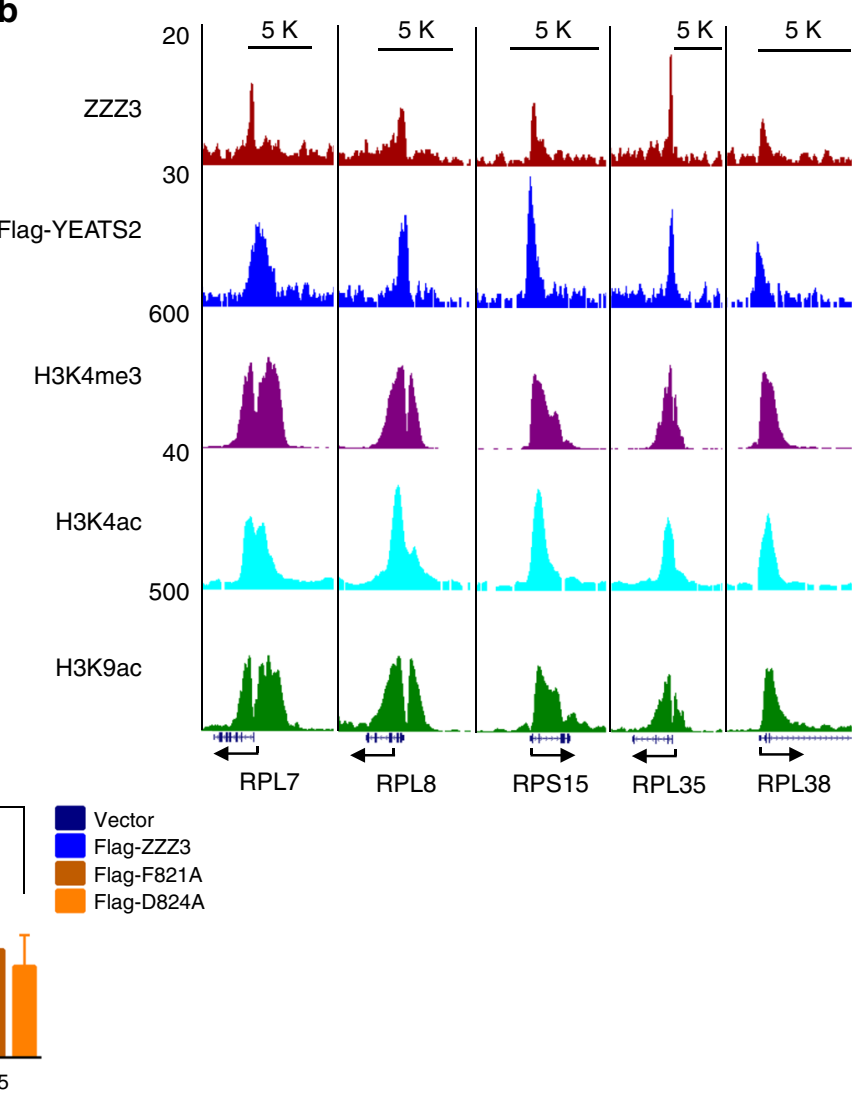

d
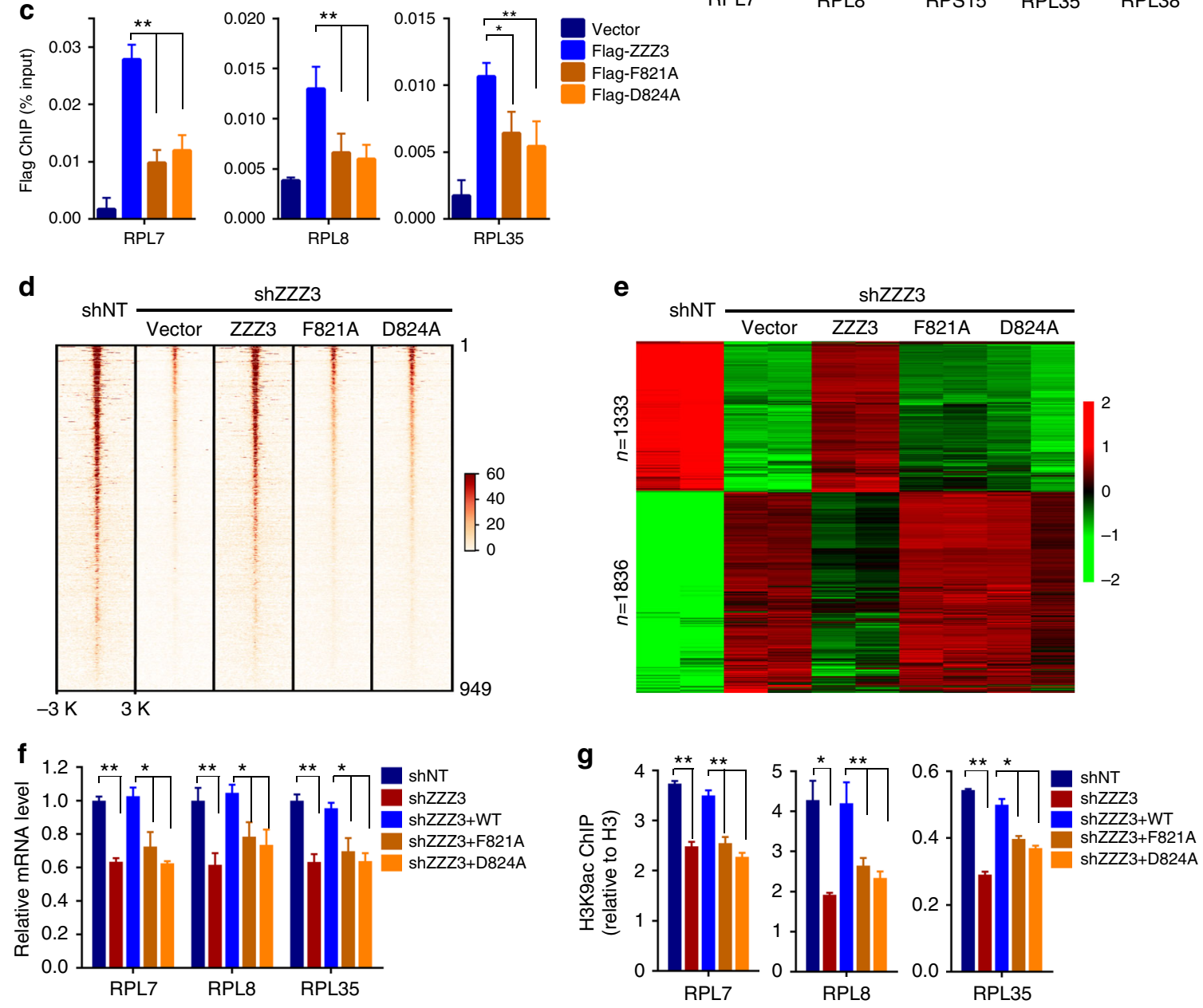

RNA-seq data uncovered 116 downregulated genes and 49 upregulated genes likely to be direct targets of ZZZ3 (Supplementary Fig. 9a and Supplementary Data 2). While the upregulated direct target genes are not significantly enriched in any pathway, the downregulated 116 genes, including 39 ribosomal protein genes, are again enriched in ribosome biogenesis (Supplementary Fig. 9b-d).

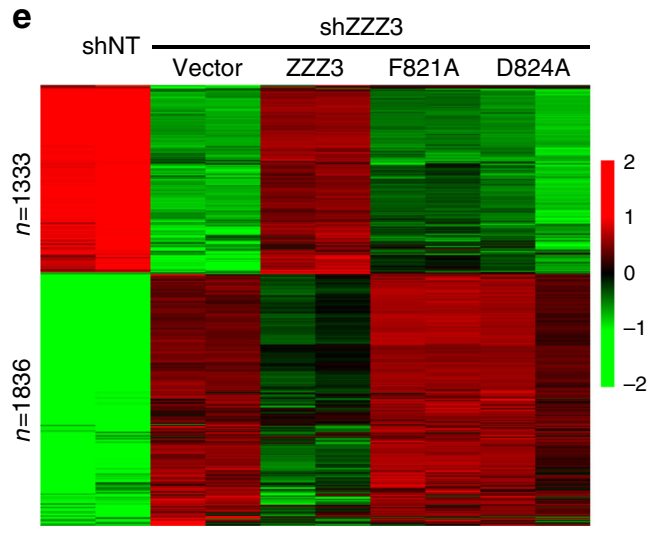

The $\mathrm{ZZ}$ domain is critical for the function of ATAC. Next, we asked whether recognition of $\mathrm{H} 3$ tail by the $\mathrm{ZZ}$ domain is required for ZZZ3 chromatin recruitment and function. To address this question, we performed "rescue" experiments by ectopically expressing Flag-tagged shRNA-resistant WT ZZZ3 or the H3-binding deficient mutants (F821A and D824A) in ZZZ3depleted H1299 cells (Supplementary Fig. 10a, b). ChIP-seq and 
Fig. 4 ZZZ3 is required for ATAC-mediated H3K9 acetylation and gene activation. a Heatmaps of the ZZZ3, Flag-YEATS2, H3K4ac, H3K9ac, and H3K4me3 ChIP-seq signals centered on ZZZ3 binding sites in a $\pm 5-k b$ window. Loci were classified as ZZZ3 peaks enriched with active promoter marks and others without active promoter marks. The color key represents the signal density. b Representative genome-browser views of ZZZ3 (red), YEATS2 (blue), H3K4me3 (purple), H3K4ac (cyan), and H3K9ac (green) ChIP-seq signals. TSS is indicated by an arrow. c qPCR analysis of Flag-ZZZ3 ChIP on gene promoters in cells stably expressing Flag-tagged WT ZZZ3 or the indicated point mutants. d Heatmap of the normalized ZZZ3 ChIP-seq signal densities in control (shNT), ZZZ3 KD (shZZZ3) cells, and KD cells ectopically expressing shRNA-resistant WT ZZZ3 or the indicated mutants. The signal is centered on $Z Z Z 3$ binding site in a $\pm 3-k b$ window. e Heatmap representation of differentially expressed genes in cells as in $\mathbf{d}$ from two biological replicates of RNA-seq experiments. Fisher's exact test was used to define differentially expressed genes $(q<0.01)$. The color key represents normalized Log2 expression values. $\mathbf{f}$ qRT-PCR analysis of the expression of the indicated ribosomal protein genes in control (shNT), ZZZ3 KD (shZZZ3) cells, and KD cells ectopically expressing shRNA-resistant WT ZZZ3 or the indicated mutants. $\mathbf{g}$ qPCR analysis of H3K9ac ChIP on a promoter of the indicated ribosomal protein genes in cells as in f. Error bars represent s.e.m. of three biological replicates. ${ }^{*} p<0.05$; ${ }^{* *} p<0.01$ (two-tailed unpaired Student's $t$-test)

ChIP-qPCR using the anti-Flag M2 antibody revealed that substituting alanine for either F821 or D824 resulted in reduced ZZZ3 occupancy at target gene promoters (Fig. 4c, Supplementary Fig. 10c, d, and Supplementary Data 1). ChIP-seq experiments using the ZZZ3-specific antibody demonstrated that introducing WT ZZZ3, but not the F821A or D824A mutant, into the ZZZ3 KD cells restored genomic distribution of ZZZ3 (Fig. 4d, Supplementary Fig. 10e and Supplementary Data 1). Finally, transcriptional profiling analyses revealed that the $\mathrm{H} 3-$ binding deficient mutants failed to rescue global transcriptional changes of the ZZZ3 KD cells (Fig. 4e and Supplementary Data 2). The failure of the H3-binding deficient mutants of ZZZ3 in restoring target gene expression (Fig. 4f) was likely attributed to the defect of the ZZZ3 mutants associated ATAC complex in acetylating histones on target gene promoters (Fig. $4 \mathrm{~g}$ ).

In summary, we identified a new reader of histone $\mathrm{H} 3$ with a recognition mode distinct from all known histone $\mathrm{H} 3$ readers. The ZZ-H3 interaction is important for the recruitment of ZZZ3 and the ATAC complex to maintain an acetylated, open chromatin environment, thus promoting gene activation. Notably, TADA2A and TADA2B components of the ATAC and SAGA complexes, respectively, also contain a $\mathrm{ZZ}$ module, but do not bind to histone $\mathrm{H} 3$. In future studies, it will be interesting to determine the functions of these $\mathrm{ZZ}$ domains and how they have evolved to perform functions distinct from recognizing histone H3.

\section{Methods}

Materials. Human ZZZ3 cDNA (NCBI Gene ID 26009) was cloned into pENTR3C, and subsequently cloned into p3FLAG, pCDH-Flag destination vectors using Gateway techniques (Invitrogen). The cDNA encoding the $\mathrm{ZZ}$ domain (amino acids 800-903) of human ZZZ3 was cloned into the pGEX-6P1 vector (Novagen). Point mutations were generated using a site-directed mutagenesis kit (Stratagene). Histone peptides bearing different modifications were synthesized at the CPC and Scilight Biotechnology. Purified recombinant mononucleosomes were purchased from EpiCypher, Inc. The anti-SGF29 and ChIP-seq-grade ZZZ3 antibodies were provided by Dr. Laszlo Tora ${ }^{26}$. Anti-histone antibodies (with dilution for western blotting) including anti-H3 (Ab1791, 1:20,000), anti-H3K9ac (Ab32129, 1:1000), anti-H3K14ac (Ab52946, 1:1000), anti-H4 (Ab7311, 1:1000), anti-H2A (Ab18255, 1:1000), anti-H2B (Ab52599, 1:1000), and anti-H3K9me3 (Ab8898, 1:1000) antibodies were obtained from Abcam. Anti-GCN5 (sc-20698, 1:2000), anti-PCAF (sc-13124, 1:200), anti-ADA3 (sc-98821, 1:1000), anti-ATAC2 (sc-398475, 1:1000), and anti-GST (sc-459, 1:1000) antibodies were from Santa Cruz. Anti-ZZZ3 (SAB4501106, 1:1000), anti-Flag (M2, 1:5000), and anti-tubulin (T8328, 1:5000) antibodies were from Sigma. Anti-H3K9ac (61251) and antiH3K4ac (39381) for ChIP-seq were from Active Motif. The shRNAs targeting human ZZZ3 were obtained from Sigma. shRNA sequences were ZZZ3\#1, 5' -AT CGTATGGGACCAATATAC; and ZZZ3\#2, 5'-GCATCAGATGACGAAAGTA TT.

Peptide microarray, peptide, and GST pulldown assays. Peptide microarray and peptide pulldown assays were performed as described previously ${ }^{27}$. Briefly, biotinylated histone peptides were printed in triplicate onto a streptavidin-coated slide (PolyAn) using a VersArray Compact Microarrayer (Bio-Rad). After a short blocking with biotin (Sigma), the slides were incubated with the GST-fused ZZZ3 $\mathrm{ZZ}$ or other desired proteins in binding buffer $(50 \mathrm{mM}$ Tris- $\mathrm{HCl} 7.5,300 \mathrm{mM}$ $\mathrm{NaCl}, 0.1 \% \mathrm{NP}-40,1 \mathrm{mM} \mathrm{PMSF}$, and $20 \%$ fetal bovine serum) overnight at $4{ }^{\circ} \mathrm{C}$ with gentle agitation. After being washed with the same buffer, the slides were probed with an anti-GST primary antibody and then a fluorescein-conjugated secondary antibody and visualized using a GenePix 4000 scanner (Molecular Devices).

For the peptide pulldown assays, $1 \mu \mathrm{g}$ of biotinylated histone peptides with different modifications were incubated with 1-2 $\mu \mathrm{g}$ of GST-fused proteins in binding buffer (50 mM Tris- $\mathrm{HCl} 7.5,300 \mathrm{mM} \mathrm{NaCl}, 0.1 \% \mathrm{NP}-40$, and $1 \mathrm{mM}$ PMSF) overnight. Streptavidin beads (Amersham) were added to the mixture, and the mixture was incubated for $1 \mathrm{~h}$ with rotation. The beads were then washed three times and analyzed using SDS-PAGE and western blotting.

For GST pull-down experiments, $2 \mu \mathrm{g}$ of GST-fused proteins were incubated with $10 \mu \mathrm{g}$ of calf thymus total histones (Worthington) in binding buffer ( $50 \mathrm{mM}$ Tris- $\mathrm{HCl} 7.5,1 \mathrm{M} \mathrm{NaCl}, 1 \% \mathrm{NP}-40,0.5 \mathrm{mM}$ EDTA, and $1 \mathrm{mM}$ PMSF plus protease inhibitors (Roche)) at $4{ }^{\circ} \mathrm{C}$ overnight, followed by an additional $1 \mathrm{~h}$ of GlutathioneSepharose beads (Amersham) incubation. The beads were then washed five times and analyzed using SDS-PAGE and western blotting. Other steps are essentially the same as described above.

Protein expression and purification. The human ZZZ3 ZZ domain (aa 816-874 and 800-903) was cloned into a pGEX 6p-1 vector and expressed in BL21 (DE3) RIL cells. Protein production was induced with $0.2 \mathrm{mM}$ IPTG and incubated overnight at $18^{\circ} \mathrm{C}$ in Luria broth (LB) or minimal media (M9) supplemented with $0.05 \mathrm{mM} \mathrm{ZnCl}_{2}$. For the production of ${ }^{13} \mathrm{C} /{ }^{15} \mathrm{~N}$-labeled or ${ }^{15} \mathrm{~N}$-labeled samples, ${ }^{15} \mathrm{NH}_{4} \mathrm{Cl}$ and ${ }^{13} \mathrm{C}_{6}$-glucose were used in the M9 medium. The GST-ZZ proteins were purified on glutathione Sepharose 4B beads (GE Healthcare) in $20 \mathrm{mM}$ Tris- $\mathrm{HCl}$ ( $\mathrm{pH} 7.0$ ) buffer, supplemented with $100 \mathrm{mM} \mathrm{NaCl}$ and $5 \mathrm{mM}$ DTT. The GST tag was cleaved overnight at $4{ }^{\circ} \mathrm{C}$ with PreScission protease. Unlabeled and ${ }^{13} \mathrm{C} /{ }^{15} \mathrm{~N}$-labeled proteins were further purified by size-exclusion chromatography and concentrated in Millipore concentrators. All mutants were generated by sitedirected mutagenesis using the Stratagene QuikChange mutagenesis protocol, grown, and purified as WT proteins.

NMR experiments. NMR experiments were carried out at $298 \mathrm{~K}$ on Varian INOVA $600-$ and $900-\mathrm{MHz}$ spectrometers. NMR samples contained $20 \mathrm{mM}$ Tris-HCl (pH 7.0) buffer, supplemented with $100 \mathrm{mM} \mathrm{NaCl}, 5 \mathrm{mM} \mathrm{DTT}$, and $8 \%$ $\mathrm{D} 2 \mathrm{O}$. The chemical shift assignments for apo-state were obtained by a set of tripleresonance experiments ${ }^{28}$ with nonlinear sampling ${ }^{29}$ using a ${ }^{13} \mathrm{C} /{ }^{15} \mathrm{~N}$-labeled ZZZ3 $_{816-874}$ sample. To obtain the H3- or H3K4ac-bound state, 10 mM H3 (1-12) peptide or $4 \mathrm{mM} \mathrm{H} 3 \mathrm{~K} 4 \mathrm{ac}(1-8)$ was added to $2 \mathrm{mM}{ }^{13} \mathrm{C} /{ }^{15} \mathrm{~N}$-labeled $\mathrm{ZZZ3}_{816-874}$ sample. The concentration of peptides was calculated by weighing $\sim 1 \mathrm{mg}$ of each peptide and dissolving in water to obtain $50-100 \mathrm{mM}$ stock solutions. The stock solutions were prepared independently for the NMR and fluorescence experiments Triple-resonance experiments were collected to confirm the assignments for the complex state. $3 \mathrm{D}{ }^{15} \mathrm{~N}$ - and ${ }^{13} \mathrm{C}$-edited NOESY-HSQC spectra (mixing time of $100 \mathrm{~ms}$ ) were collected to obtain distance restraints within ZZZ3-ZZ. $2 \mathrm{D}^{15} \mathrm{~N}-/{ }^{13} \mathrm{C}-$ filtered NOESY and TOCSY-HSQC spectra were collected to obtain the chemical shift assignment for the $\mathrm{H} 3$ peptide. $3 \mathrm{D}{ }^{15} \mathrm{~N}$-/ ${ }^{13} \mathrm{C}$-filtered ${ }^{13} \mathrm{C}$ - and ${ }^{15} \mathrm{~N}$-edited NOESY-HSQC spectra (mixing time of 200 and $300 \mathrm{~ms}$ for H3 (1-12), and of 200 $\mathrm{ms}$ for H3K4ac (1-8)) were collected to obtain the intermolecular distance restraints.

In total, $0.1 \mathrm{mM}$ uniformly ${ }^{15} \mathrm{~N}$-labeled WT or mutated ZZZ3-ZZ was used in NMR titrations. Binding was characterized by monitoring chemical shift changes in the proteins induced by histone peptides (synthesized by SynPeptide). Dissociation constant $\left(K_{\mathrm{d}}\right)$ was determined by applying PCA to the ${ }^{1} \mathrm{H},{ }^{15} \mathrm{~N}$ HSQC titration spectra in TREND ${ }^{30}$. Each binding isotherm was fitted using the following equation:

$$
p_{\text {bound }}=\|P C 1\|=\frac{\left(\left([L]+[P]+K_{\mathrm{d}}\right)-\sqrt{\left.\left([L]+[P]+K_{\mathrm{d}}\right)^{2}-4[P][L]\right)}\right)}{2[P]}
$$

where $[L]$ is the concentration of the histone peptide, $[P]$ is the concentration of $\mathrm{ZZ}, p_{\text {bound }}$ is the fraction of protein bound to a ligand, and $P C 1$ is the normalized 
principal component, obtained by TREND, that indicates the change in the population of the bound state. The errors of the $K_{\mathrm{d}}$ value are the fitting uncertainties from nonlinear least-squares fits in Kaleidagraph.

Structure determination for the ZZZ3-H3 complex. Calculation of the structure of ZZZ3-ZZ (aa 816-874) in complex with H3 (1-12) peptide or H3K4ac (1-8) peptide was carried out using interproton NOE-derived distance restraints and dihedral angle restraints. Spectra were processed and analyzed with NMRDraw and CcpNmr Suite ${ }^{31}$. The program DANGLE in CcpNmr Suite was used to predict dihedral angles $\psi$ and $\varphi$ restraints. Hydrogen bonds were derived from characteristic NOE patterns in combination with dihedral angles. The structures were calculated initially with XPLOR-NIH and refined with AMBER ${ }^{32,33}$. Hundred structures were calculated, and the ensemble of 20 conformers with the lowest total energy was selected to represent the complex of ZZZ3-ZZ and H3 peptides. The quality of the structures was validated using the program PROCHECK-NMR. The structural statistics is listed in Supplementary Table 1.

Fluorescence spectroscopy. Spectra were recorded at $25^{\circ} \mathrm{C}$ on a Fluoromax-3 spectrofluorometer (HORIBA). The samples containing $1.0 \mu \mathrm{M} \mathrm{ZZZ3} \mathrm{ZZ} \mathrm{(aa}$ $800-903$ ) and progressively increasing concentrations of the histone peptide were excited at $295 \mathrm{~nm}$. Experiments were performed in buffer containing $20 \mathrm{mM}$ Tris- $\mathrm{HCl}(\mathrm{pH} 7.2), 150 \mathrm{mM} \mathrm{NaCl}$, and $1 \mathrm{mM}$ DTT, with the exception of experiments shown in Supplementary Fig. 3b, which were performed in $100 \mathrm{mM}$ $\mathrm{NaCl}$ buffer. Emission spectra were recorded over a range of wavelengths between 330 and $360 \mathrm{~nm}$ with a $0.5-\mathrm{nm}$ step size and a 1 -s integration time and averaged over three scans. The $K_{\mathrm{d}}$ values were determined using a nonlinear least-squares analysis and the equation:

$$
\Delta I=\Delta I_{\max } \frac{\left(\left([L]+[P]+K_{\mathrm{d}}\right)-\sqrt{\left([L]+[P]+K_{\mathrm{d}}\right)^{2}-4[P][L]}\right)}{2[P]}
$$

where $[L]$ is the concentration of the histone peptide, $[P]$ is the concentration of ZZZ3 ZZ domain, $\Delta I$ is the observed change of signal intensity, and $\Delta I_{\max }$ is the difference in signal intensity of the free and bound states of the ZZ domain. The $K_{\mathrm{d}}$ value was averaged over three separate experiments, with error calculated as the standard deviation between the runs.

Microscale thermophoresis (MST) binding assay. The MST experiments were performed using a Monolith NT.115 instrument (NanoTemper) as described previously ${ }^{34}$. All experiments were performed with the purified $\mathrm{ZZ}$ domain (a.a. $800-903)$ in a buffer containing $20 \mathrm{mM}$ Tris- $\mathrm{HCl}(\mathrm{pH} 7.0), 150 \mathrm{mM} \mathrm{NaCl}$, and 3 mM DTT. The final concentration of the C-terminal fluorescein-labeled histone $\mathrm{H} 3$ peptide (1-12, KE BIOCHEM) was kept at $80 \mathrm{nM}$. Dissociation constants for the interaction between ZZ with unlabeled peptides H3 (1-12) and H3K4ac (1-12) were measured using a displacement assay in which increasing amounts of unlabeled peptides were added into a preformed ZZ:H3-FAM complex prepared by supplementing $5 \mu \mathrm{M} \mathrm{ZZ} \mathrm{into} \mathrm{each} \mathrm{sample.} \mathrm{The} \mathrm{measurements} \mathrm{were} \mathrm{performed} \mathrm{at}$ $50 \%$ LED and $40 \%$ MST power with 3-s laser on time and $22 \mathrm{~s}$ off time. For all measurements, samples were loaded into premium capillaries and 1400-1700 counts were obtained for the fluorescence intensity. The $K_{\mathrm{d}}$ and $\mathrm{IC}_{50}$ values were determined with the MO.Affinity Analysis software (NanoTemper Technologies $\mathrm{GmbH})$, using two independent MST measurements. The $K_{\mathrm{i}}$ values for unlabeled peptides with $\mathrm{ZZ}$ were determined from the $\mathrm{IC}_{50}$ values observed in the displacement assay and converted by the following equation:

$$
K_{\mathrm{i}}=[I]_{50} /\left(\frac{[L]_{50}}{K_{\mathrm{d}}}+\frac{[P]_{0}}{K_{\mathrm{d}}}+1\right)
$$

where $[\mathrm{I}]_{50}$ is the concentration of free unlabeled ligand at $50 \%$ binding and $[\mathrm{L}]_{50}$ is the concentration of free labeled $\mathrm{H} 3$ peptide at $50 \%$ binding. The $K_{\mathrm{d}}$ value is the dissociation constant of labeled $\mathrm{H} 3$ peptide determined in the direct binding experiment described above. Measurements for $\mathrm{H} 3$ unmodified and $\mathrm{H} 3 \mathrm{~K} 4 \mathrm{ac}$ peptides were done in triplicates and duplicates, respectively.

Affinity purification and HAT assays. Nuclear extracts were prepared from stable cells using a standard protocol ${ }^{35}$. Briefly, approximately $2 \mathrm{mg}$ of nuclear extract was incubated with $25 \mu \mathrm{l}$ of M2-agarose beads (Sigma) overnight at $4{ }^{\circ} \mathrm{C}$. The beads were washed four times with high-salt wash buffer (10 mM HEPES, pH 7.9, 25\% glycerol, $1.5 \mathrm{mM} \mathrm{MgCl}_{2}, 300 \mathrm{mM} \mathrm{KCl}$, and $0.1 \%$ Triton X-100), followed by two washes with low-salt wash buffer (10 mM HEPES, pH 7.9, 25\% glycerol, $1.5 \mathrm{mM}$ $\mathrm{MgCl}_{2}, 100 \mathrm{mM} \mathrm{KCl}$, and $0.1 \%$ Triton X-100). Elution was achieved by two consecutive incubations of the beads with $0.5 \mathrm{mg} / \mathrm{ml}$ triple-FLAG peptide (Sigma) in $200 \mu \mathrm{l}$ of low-salt wash buffer. All buffers contained phenylmethylsulfonyl fluoride and protease inhibitor cocktail (Roche).

Purified complexes were incubated for indicated times at $37^{\circ} \mathrm{C}$ with peptides $(1 \mu \mathrm{g})$ or mononucleosomes $(10 \mu \mathrm{g})$ and $0.1 \mathrm{mM}$ acetyl-CoA in HAT reaction buffer (50 mM Tris, pH 8.0, $0.1 \mathrm{mM}$ EDTA, 10\% glycerol, $1 \mathrm{mM}$ PMSF, and $1 \mathrm{mM}$ DTT) in a total volume of $50 \mu \mathrm{L}$. Reactions were quenched by flash-freezing in liquid nitrogen and then analyzed by SDS-PAGE and western blot. Uncropped western blots are shown in Supplementary Figure 11.

Cell culture and viral transduction. All cell lines were validated by STR DNA fingerprinting performed by the MDACC CCSG-funded Characterized Cell Line Core (NCI \# CA016672). Human HEK 293 T (ATCC) cells were maintained in DMEM (Cellgro) supplemented with 10\% fetal bovine serum (Sigma). Human lung cancer cell lines H1299 and A549 were cultured in RPMI 1640 (Cellgro) supplemented with $10 \%$ fetal bovine serum. Lentiviral transduction was performed as described previously ${ }^{27}$. Briefly, 293T cells were co-transfected with pMD2.G, pPAX2 (Addgene) and pLKO shRNA, or pCDH cDNA constructs and control vectors, and then viral supernatants were collected after $48 \mathrm{~h}$. For infections, cells were incubated with viral supernatants in the presence of $8 \mu \mathrm{g} / \mathrm{ml}$ polybrene. After $48 \mathrm{~h}$, the infected cells were selected with puromycin $(2 \mu \mathrm{g} / \mathrm{ml})$ for pLKO clones or blasticidin $(10 \mu \mathrm{g} / \mathrm{ml})$ for $\mathrm{pCDH}$ clones for 3-4 days before experiments.

ChIP and ChIP-seq analysis. ChIP analysis was performed essentially as described previously ${ }^{27}$. Briefly, cells were cross-linked with $1 \%$ formaldehyde for $10 \mathrm{~min}$ and stopped with $125 \mathrm{mM}$ glycine. Nuclei were isolated by re-suspending the cells in swelling buffer containing $5 \mathrm{mM}$ PIPES, pH $8.0,85 \mathrm{mM} \mathrm{KCl}, 1 \% \mathrm{NP}-40$, and a complete protease inhibitor for $20 \mathrm{~min}$ at $4{ }^{\circ} \mathrm{C}$. The isolated nuclei were resuspended in nuclei lysis buffer (50 mM Tris, pH 8.0, $10 \mathrm{mM}$ EDTA, and 1\% SDS) and sonicated using a Bioruptor Sonicator (Diagenode). The samples were immunoprecipitated with $2-4 \mu \mathrm{g}$ of the appropriate antibodies overnight at $4{ }^{\circ} \mathrm{C}$. Protein A/G beads were added and incubated for $1 \mathrm{~h}$, and the immunoprecipitates were washed twice each with low-salt, high-salt, and LiCl buffers. Eluted DNA was reverse-cross-linked, purified using PCR purification kit (Qiagene), and analyzed by quantitative real-time PCR on the ABI 7500-FAST System using the Power SYBR Green PCR Master Mix (Applied Biosystems). Statistical differences were calculated using a two-way unpaired Student's $t$-test. The primers used for qPCR are listed in Supplementary Data 3.

For ChIP-seq, ChIP experiments were carried out essentially the same as described above. Samples were sequenced using the Illumina Solexa Hiseq 2500. The raw reads were mapped to human reference genome NCBI 37 (hg19) by Solexa data-processing pipeline, allowing up to two mismatches. The genome ChIP-sec profiles were generated using MACS 1.3.6 with only unique mapped reads. Clonal reads were automatically removed by MACS. The ChIP-seq profiles were normalized to $10,000,000$ total tag numbers, and peaks were called at $p$-values $\leq 1 \mathrm{e}-8$ with input DNA as control. The input DNA was also used for peak calling. Nonspecific peaks called in the input DNA were used to remove false-positive peaks from ChIP samples. For ChIP-seq peak calling, promoters are defined as [ $-3 \mathrm{k} \sim 3 \mathrm{k}]$ bp around TSSs of RefSeq genes. A binomial test was used to evaluate the significance of ChIP-seq peak occupancy (at least $1 \mathrm{nt}$ ) on promoters. ChIP-seq heatmap was drawn by the seqplots R package (http://github.com/przemol/ seqplots).

RNA extraction, real-time PCR, and RNA-seq analysis. Reverse transcription PCR and real-time PCR were performed as previously described ${ }^{27}$. Total RNA was extracted using an RNeasy plus kit (Qiagen) and reverse-transcribed using an iScrip reverse transcription kit (Bio-Rad). Quantitative real-time PCR (qPCR) analyses were performed as described previously using Power SYBR Green PCR Master Mix and the ABI 7500-FAST Sequence Detection System (Applied Biosystems). Gene expressions were calculated following normalization to GAPDH levels using the comparative $\mathrm{Ct}$ (cycle threshold) method. For glucose starvation, gene expressions were calculated following normalization to cell number using the comparative $\mathrm{Ct}$ (cycle threshold) method. Statistical differences were calculated using a two-way unpaired Student's $t$-test. The primer sequences for qPCR are listed in Supplementary Data 3.

RNA-seq samples were sequenced using the Illumina Hiseq 2500, and raw reads were mapped to the human reference genome (hg19) and transcriptome using Tophat 2.1.0 (http://ccb.jhu.edu/software/tophat/index.shtml). Read counts for each transcript were calculated using HTseq v0.6.1 using default parameters. Differential gene expression analyses were performed using the "exactTest" function in edgeR v3.0. Gene Ontology analysis was performed using the DAVID Bioinformatics Resource 6.7. The gene expression heatmap was generated using pheatmap package in CRAN (https://cran.r-project.org/package=pheatmap). The volcano plot was drawn using ggplot2 package in $\mathrm{R}$ computing environment (https://cran.r-project.org/package=ggplot2).

Statistical analyses. Experimental data are presented as mean \pm s.e.m. unless stated otherwise. Statistical significance was calculated by two-tailed unpaired $t$-test on two experimental conditions with $p<0.05$ considered statistically significant unless stated otherwise. Statistical significance levels are denoted as follows: ${ }^{*} p<0.05 ;{ }^{* *} p<0.01 ;{ }^{* * *} p<0.001$; n. s.: not significant. No statistical methods were used to predetermine sample size. For overlap analysis, a hypergeometric test was performed for a two-way Venn diagram by the R software unless otherwise stated. Super exact test was performed for the three-way Venn diagram by the R package (https://lib.ugent.be/CRAN/web/packages/SuperExactTest). 


\section{Data availability}

Structure data have been deposited in Protein Data Bank under accession numbers 6E83 and 6E86. The ChIP-seq and RNA-seq data have been deposited in the Gene Expression Omnibus database under accession number GSE100009.

Received: 22 June 2018 Accepted: 15 August 2018

Published online: 14 September 2018

\section{References}

1. Strahl, B. D. \& Allis, C. D. The language of covalent histone modifications. Nature 403, 41-45 (2000).

2. Turner, B. M. Cellular memory and the histone code. Cell 111, 285-291 (2002).

3. Li, B., Carey, M. \& Workman, J. L. The role of chromatin during transcription. Cell 128, 707-719 (2007).

4. Jenuwein, T. \& Allis, C. D. Translating the histone code. Science $\mathbf{2 9 3}$ 1074-1080 (2001).

5. Bannister, A. J. et al. Selective recognition of methylated lysine 9 on histone H3 by the HP1 chromo domain. Nature 410, 120-124 (2001).

6. Lachner, M., O'Carroll, D., Rea, S., Mechtler, K. \& Jenuwein, T. Methylation of histone H3 lysine 9 creates a binding site for HP1 proteins. Nature 410, 116-120 (2001).

7. Wysocka, J. et al. A PHD finger of NURF couples histone H3 lysine 4 trimethylation with chromatin remodelling. Nature 442, 86-90 (2006).

8. Shi, X. et al. ING2 PHD domain links histone H3 lysine 4 methylation to active gene repression. Nature 442, 96-99 (2006).

9. Dhalluin, C. et al. Structure and ligand of a histone acetyltransferase bromodomain. Nature 399, 491-496 (1999).

10. Li, Y. et al. AF9 YEATS domain links histone acetylation to DOT1L-mediated H3K79 methylation. Cell 159, 558-571 (2014).

11. Guelman, S. et al. Host cell factor and an uncharacterized SANT domain protein are stable components of ATAC, a novel dAda2A/dGcn5-containing histone acetyltransferase complex in Drosophila. Mol. Cell. Biol. 26, 871-882 (2006).

12. Wang, Y. L., Faiola, F., Xu, M. Y., Pan, S. Q. \& Martinez, E. Human ATAC Is a GCN5/PCAF-containing Acetylase Complex with a novel NC2-like histone fold module that interacts with the TATA-binding protein. J. Biol. Chem. 283 , 33808-33815 (2008).

13. Nagy, Z. et al. The metazoan ATAC and SAGA coactivator HAT complexes regulate different sets of inducible target genes. Cell. Mol. Life Sci. 67, 611-628 (2010).

14. Spedale, G., Timmers, H. T. M. \& Pijnappel, W. W. M. P. ATAC-king the complexity of SAGA during evolution. Gene Dev. 26, 527-541 (2012).

15. Lee, K. K. et al. Combinatorial depletion analysis to assemble the network architecture of the SAGA and ADA chromatin remodeling complexes. Mol. Syst. Biol. 7, 503 (2011).

16. Wang, L. \& Dent, S. Y. R. Functions of SAGA in development and disease Epigenomics-Uk 6, 329-339 (2014).

17. Couture, J. F., Collazo, E. \& Trievel, R. C. Molecular recognition of histone H3 by the WD40 protein WDR5. Nat. Struct. Mol. Biol. 13, 698-703 (2006)

18. Ruthenburg, A. J. et al. Histone $\mathrm{H} 3$ recognition and presentation by the WDR5 module of the MLL1 complex. Nat. Struct. Mol. Biol. 13, 704-712 (2006).

19. Klein, B. J. et al. The histone-H3K4-specific demethylase KDM5B binds to its substrate and product through distinct PHD fingers. Cell Rep. 6, 325-335 (2014).

20. Xiong, X. Z. et al. Selective recognition of Histone crotonylation by double PHD fingers of MOZ and DPF2. Nat. Chem. Biol. 12, 1111-+ (2016).

21. Ali, M. et al. Tandem PHD fingers of MORF/MOZ acetyltransferases display selectivity for acetylated Histone $\mathrm{H} 3$ and are required for the association with chromatin. J. Mol. Biol. 424, 328-338 (2012).

22. Zeng, L. et al. Mechanism and regulation of acetylated histone binding by the tandem PHD finger of DPF3b. Nature 466, 258-262 (2010).

23. Li, W. G., Zhao, A., Tempel, W., Loppnau, P. \& Liu, Y. L. Crystal structure of DPF3b in complex with an acetylated histone peptide. J. Struct. Biol. 195, 365-372 (2016).

24. Mansfield, R. E. et al. Plant homeodomain (PHD) fingers of CHD4 are Histone H3-binding modules with preference for unmodified H3K4 and methylated H3K9. J. Biol. Chem. 286, 11779-11791 (2011).

25. Guillemette, B. et al. H3 lysine 4 is acetylated at active gene promoters and is regulated by $\mathrm{H} 3$ lysine 4 methylation. PLoS. Genet. 7, doi:ARTN e100135410.1371/journal.pgen.1001354 (2011).

26. Krebs, A. R., Karmodiya, K., Lindahl-Allen, M., Struhl, K. \& Tora, L. SAGA and ATAC histone acetyl transferase complexes regulate distinct sets of genes and ATAC defines a class of p300-independent enhancers. Mol. Cell 44, 410-423 (2011).

27. Wen, H. et al. ZMYND11 links histone H3.3K36me3 to transcription elongation and tumour suppression. Nature 508, 263-268 (2014).

28. Sattler, M., Schleucher, J. \& Griesinger, C. Heteronuclear multidimensional NMR experiments for the structure determination of proteins in solution employing pulsed field gradients. Prog. Nucl. Mag. Res Sp. 34, 93-158 (1999).

29. Hyberts, S. G., Milbradt, A. G., Wagner, A. B., Arthanari, H. \& Wagner G. Application of iterative soft thresholding for fast reconstruction of NMR data non-uniformly sampled with multidimensional Poisson Gap scheduling. J. Biomol. NMR 52, 315-327 (2012).

30. $\mathrm{Xu}, \mathrm{J}$. \& Van Doren, S. R. Binding isotherms and time courses readily from magnetic resonance. Anal. Chem. 88, 8172-8178 (2016).

31. Vranken, W. F. et al. The CCPN data model for NMR spectroscopy: development of a software pipeline. Proteins 59, 687-696 (2005).

32. Schwieters, C. D., Kuszewski, J. J., Tjandra, N. \& Clore, G. M. The Xplor-NIH NMR molecular structure determination package. J. Magn. Reson. 160, 65-73 (2003).

33. Case, D. A. et al. The Amber biomolecular simulation programs. J. Comput. Chem. 26, 1668-1688 (2005).

34. Wienken, C. J., Baaske, P., Rothbauer, U., Braun, D. \& Duhr, S. Proteinbinding assays in biological liquids using microscale thermophoresis. Nat. Commun. 1, 100 (2010).

35. Abmayr, S. M., Yao, T., Parmely, T. \& Workman, J. L. Preparation of nuclear and cytoplasmic extracts from mammalian cells. Curr. Protoc. Mol. Biol. Chapter 12, 10.1002/0471142727.mb1201s75 (2006)

\section{Acknowledgements}

We thank M. Bedford, S.Y.R. Dent, B. Gan, J. Kurie, and H. Lee for sharing reagents and scientific discussions. We thank B. Dennehey for editing the manuscript. We thank the MD Anderson Science Park Next-Generation Sequencing Facility (CPRIT RP120348) for Solexa sequencing. This work was supported in part by grants from NIH/NCI (CA204020), Cancer Prevention and Research Institute of Texas (RP160237 and RP160739), and Welch Foundation (G1719) to X.S., NIH (GM106416, GM125195, and GM100907) to T.G.K., and CPRIT (RP150292) and NIH (HG007538 and CA193466) to W.L. X.S. is a Leukemia \& Lymphoma Society Career Development Program Scholar.

\section{Author contributions}

W.M., Y.Z., and J.L. contributed equally to this work. X.S., T.K., W.M., and Y.Z. conceived the study. W.M. performed the biochemical and cellular studies; Y.Z. performed the structural, NMR, and fluorescence studies with help from Q.T. and A.H.T; J.L. performed bioinformatics analysis; X.W., D.P., and Y.X provided technical assistance; X.S., T.K., W.M., and Y.Z. wrote the paper with comments from H.W., J.L., and W.L.

\section{Additional information}

Supplementary Information accompanies this paper at https://doi.org/10.1038/s41467018-06247-5.

Competing interests: X.S. is a Scientific Advisory Board member of EpiCypher. All other authors declare no competing interests.

Reprints and permission information is available online at http://npg.nature.com/ reprintsandpermissions/

Publisher's note: Springer Nature remains neutral with regard to jurisdictional claims in published maps and institutional affiliations.

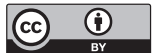

Open Access This article is licensed under a Creative Commons Attribution 4.0 International License, which permits use, sharing adaptation, distribution and reproduction in any medium or format, as long as you give appropriate credit to the original author(s) and the source, provide a link to the Creative Commons license, and indicate if changes were made. The images or other third party material in this article are included in the article's Creative Commons license, unless indicated otherwise in a credit line to the material. If material is not included in the article's Creative Commons license and your intended use is not permitted by statutory regulation or exceeds the permitted use, you will need to obtain permission directly from the copyright holder. To view a copy of this license, visit http://creativecommons.org/ licenses/by/4.0/.

(C) The Author(s) 2018 